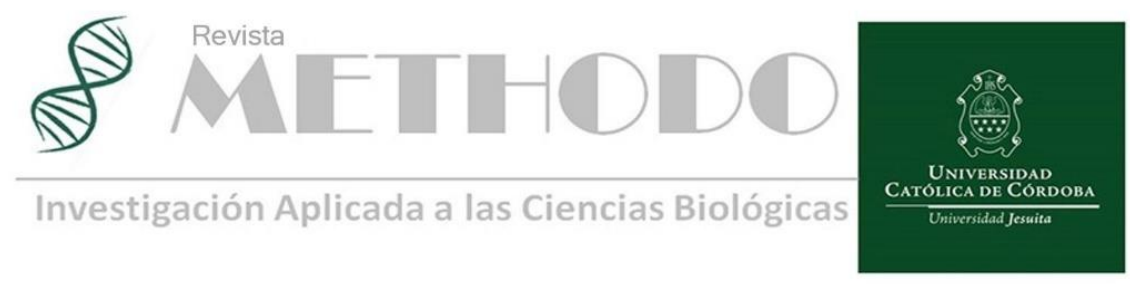

SEMBLANZA Rev. Methodo 2021;6(4):199-200 https://doi.org/10.22529/me.2021.6(4)09 Recibido 01 Jun. 2021 | Publicado 05 Oct. 2021

\title{
Prof. Juan Gastón René Durigneux (1924 -1997)
}

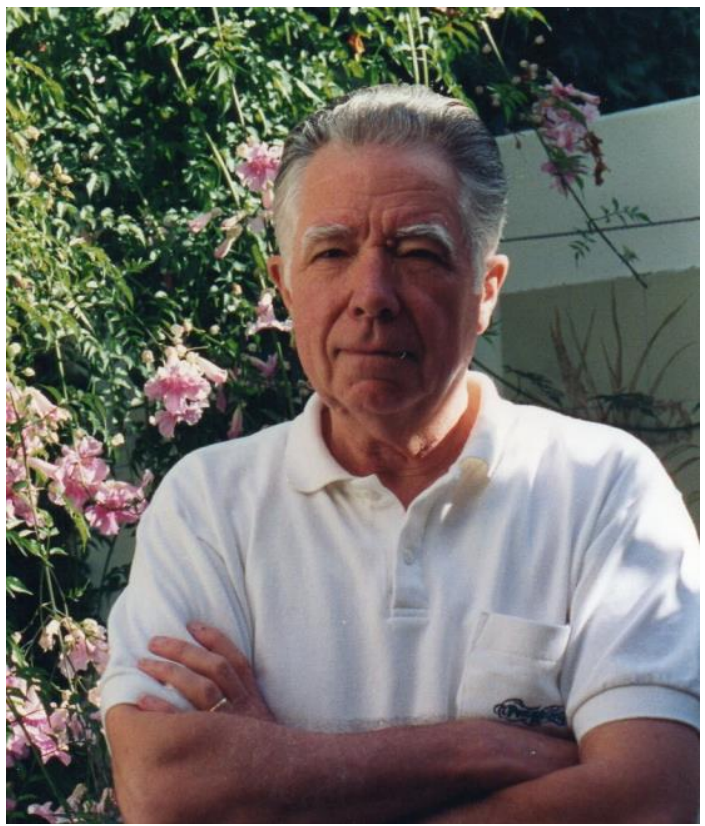

Figura 1. Prof. Juan Gastón René Durigneux

\section{Un grifo que vertió una historia}

Era una hermosa y fresca mañana en la Villa de Carlos Paz, aquél día del año 1959.

Un grifo que entregaba agua limpia proveniente de una vertiente cercana, instalado por los padres jesuitas en la Quinta del Niño Dios de Carlos Paz, hoy Parque Estancia La Quinta, para los vecinos del lugar, pues la Villa no poseía agua potable, fue el responsable de esta larga y conmovedora historia.
Seguramente aquel grifo jamás sospechó que llegarían con su cántaro Joseph Capel junto a su esposa Rose Bauwens y se encontrarían con un señor de ropaje negro y largo, el Padre Jean Sonet sj en aquel entonces, profesor y vicerrector de la Universidad Católica de Córdoba.

Todo comienza en Bélgica, de donde los tres nuevos amigos resultaron oriundos y que la guerra determinara que debían emigrar de su país natal, para hallar refugio y paz. Joseph y Rose, partieron en 1951 hacia Uruguay en América del Sur, con su hija Micheline Capel y su reciente esposo Juan Gastón René Durigneux, nacido en el pueblo de Mons. - (Bélgica) el 14 de abril de 1924 que se perfilaba como un joven amante de las ciencias. Así obtuvo en la Universidad Libre de Bruselas, su título profesional de Farmacéutico. Su gran vocación asociada con su inteligencia, acompañaron a este flamante profesional a una nueva aventura en tierras lejanas.

Al llegar a Montevideo, fue acogido en el Liceo Francés como docente en Matemática, Física y Química en francés, muy lejos del español local que con los años iría aprendiendo y asimilando como propio.

Joseph y Rose relataron al Padre Jean Sonet sj, que habían dejado en Uruguay a su hija, junto a su esposo y la pequeña Brigitte, su primera nietita, y que sería maravilloso que la vida los volviera a reunir.

Así fue, como el Padre Jean Sonet sj invita al joven Juan Gastón René Durigneux, a integrarse al cuerpo docente de la UCC, y su primer nombramiento fue en marzo del año 1960, como Profesor Encargado y Jefe de Trabajos Prácticos de las asignaturas Química Orgánica I y II; Analítica I

Revista Methodo: Investigación Aplicada a las Ciencias Biológicas. Universidad Católica de Córdoba. Jacinto Ríos 571 Bo Gral. Paz. X5004FXS. Córdoba. Argentina. Tel.: (54) 351 4517299 / Correo: methodo@ucc.edu.ar / Web: methodo.ucc.edu.ar | SEMBLANZA Rev. Methodo 2021;6(4):199-200. 
y II; y Análisis Instrumental, para bioquímicos y farmacéuticos. Además, de todos estos cargos asumió otro rol superior, el de padre de Verónica, su segunda hija. En 1966 es nombrado Profesor Titular de las asignaturas antes mencionadas y años después, de Química Analítica Aplicada para Licenciados en Química Industrial.

Rápidamente, el Padre Jean Sonet sj, descubre en Juan Gastón René Durigneux, valores, convicciones, metodología, exigencia para consigo mismo y en las aulas. Por todo ello lo convoca a participar de la AGCD, Agencia Gubernamental de Cooperación para el Desarrollo. Ente que, a lo largo del tiempo, incorporó a otros profesores de origen belga a la UCC, entre ellos, el Prof. Pierre Hick.

La historia sigue fluyendo y en 1972 fue nombrado Vicedecano y desde 1977 al 1985, Decano de la Facultad de Ciencias Químicas.

Cabe destacar su incorporación a la Facultad de Medicina durante los años 1974-1977, como Profesor Titular Interino en la asignatura Química Médica, actividad que le permitió brindar grandes aportes a la medicina desde la Química Analítica.

Sus ojos azules y su guardapolvo blanco portaban los colores de nuestra bandera argentina, tierra que asumió como su patria y que, con fidelidad insobornable, lo ancló para siempre sin escuchar tentadoras ofertas para seguir otros rumbos.

Era un científico nato. Su pasión por la docencia, la investigación y la UCC hacía que sus días le resultaran cortos, por eso, siempre fue el primero en llegar y el último en retirarse de la Facultad.

De sus trabajos, se obtuvieron valiosos resultados como, por ejemplo:

- Estudio de un método rápido y preciso de determinación de talio en orina y otros medios biológicos.

- Uso de una fórmula semielemental en niños con daño en la mucosa intestinal, en asociación con la Facultad de Medicina.

-Estudio sobre la salinidad de la laguna de Mar Chiquita, antes de la gran inundación que años más tarde sobrevendrá.

En el año 1977, quien escribe esta semblanza ingresaba a tercer año de la Carrera de Farmacia, Facultad de Ciencias Químicas, sabiendo que debía cursar una de las asignaturas medulares para mi carrera: Química Analítica I y II, no eran materias fáciles ni mucho menos, pero me dieron la oportunidad de conocer a lo largo del año a uno de los grandes maestros de mi vida, el Profesor Juan Gastón René Durigneux, quien con su magia y sabiduría lograba transformar una asignatura altamente exigente y complicada, en conocimientos útiles y fáciles de comprender.

Años después, un llamado del entonces Rector Miguel Ángel Moreno sj me pide hacerme cargo de dos cátedras. Coincidentemente, Química Analítica I y II, las cátedras que con tanto esfuerzo había cursado y disfrutado con el Profesor Juan Gastón René Durigneux, estaban, ahora, bajo mi responsabilidad y que desde ese momento hasta la actualidad soy, con mucho orgullo, su titular; y en las que trato con esmero de plasmar la sabiduría, compromiso y valores que he heredado del gran maestro.

Como el grifo de la historia, el agua siguió corriendo, pero esta vez desde dentro de las cátedras heredadas. En mis primeros años a cargo de magnánima responsabilidad, dictar estas asignaturas, era verlo a mi Maestro en cada mesada, en el droguero, en cada instrumento. Pero fundamentalmente era verlo con su guardapolvo blanco frente al pizarrón del laboratorio, que en su honor hoy lleva su nombre, frente a sus alumnos que con una mezcla de terror y admiración aprendíamos de su ciencia y pasión por transmitir conocimientos relacionados con la Química.

Como ex alumna y como docente, quisimos con el equipo de cátedra, tomar la bandera de su trabajo en homenaje a su dedicación y entrega, y nos propusimos escribir dos libros que recopilan información ordenada y contenida en sus manuscritos durante años (apuntes). Así pues, desde el año 2010 el "Manual de Identificación de Iones Frecuentes" y el libro de "Preparación de Reactivos para Uso Analíticos", dan fe de tal labor. Ambos se utilizan en las aulas de distintas facultades y laboratorios portando la herencia del Profesor Juan Gastón René Durigneux: su excelencia como maestro, su pasión como investigador y su compromiso ético como persona.

Una historia que comenzó en un grifo que aún ... sigue vertiendo futuro en nuestras aulas.

Prof. Adriana Beatriz Welter

(Universidad Católica de Córdoba, Facultad de Ciencias

Químicas).

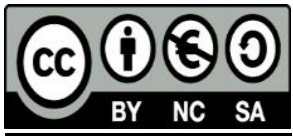

Revista Methodo: Investigación Aplicada a las Ciencias Biológicas. Universidad Católica de Córdoba. Jacinto Ríos 571 Bo Gral. Paz. X5004FXS. Córdoba. Argentina. Tel.: (54) 351 4517299 / Correo: methodo@ucc.edu.ar / Web: methodo.ucc.edu.ar | SEMBLANZA Rev. Methodo 2021;6(4):199-200. 